\title{
GMR
}

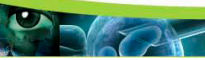

\section{miR-124 radiosensitizes human esophageal cancer cell TE-1 by targeting CDK4}

\author{
Y.H. Zhang ${ }^{1,2 *}$, Q.Q. Wang ${ }^{1,2 *}$, H. Li ${ }^{3}$, T. Ye ${ }^{3}$, F. Gao ${ }^{2}$ and Y.C. Liu ${ }^{2}$ \\ ${ }^{1}$ Bengbu Medical School, Bengbu, China \\ ${ }^{2}$ Department of Radiotherapy, Taixing People's Hospital, Taixing, China \\ ${ }^{3}$ Department of Clinical Laboratory, Taixing People's Hospital, Taixing, China \\ *These authors contributed equally to this study. \\ Corresponding author: Y.C. Liu \\ E-mail: yangchen_liutx@163.com \\ Genet. Mol. Res. 15 (2): gmr.15027893 \\ Received October 22, 2015 \\ Accepted December 23, 2015 \\ Published June 3, 2016 \\ DOI http://dx.doi.org/10.4238/gmr.15027893
}

\begin{abstract}
Radiotherapy is one of the most important treatments for esophageal cancer, but radioresistance remains a major challenge. Previous studies have shown that microRNAs (miRNAs or miRs) are involved in human cancers. miR-124 has been widely reported in various cancers and it is intimately involved in proliferation, cell cycle regulation, apoptosis, migration, and invasion of cancer cells. The aim of this study was to explore the relationship between the miR-124/cyclindependent kinase 4 (CDK4) axis and the radiosensitivity of esophageal cancer cells. In this study, we identified the reduced expression of miR124 in 18 paired esophageal cancer tissues compared to their matched normal tissues. In order to investigate the physiological role of miR124 in esophageal cancer, the cell counting kit-8 (CCK-8) assay and wound healing assay were performed, and the results suggest that miR124 overexpression decreases tumor growth and aggression. Next, we detected the effects of ectopic miR-124 expression on the apoptosis of an esophageal cancer cell line (TE-1) following radiotherapy. Using the CCK-8 assay and Hoechst 332528 stain, we found that ectopic expression of miR-124 led to a higher percentage of apoptotic cells.
\end{abstract}


Finally, we identified that CDK4 is a direct target of miR-124 in TE-1 cells using target prediction algorithms and a luciferase reporter assay. Moreover, western blot assay confirmed that CDK4 was downregulated during miR-124 transfection. Taken together, we illustrate that the miR-124/CDK4 axis plays an important role in radiation sensitivity of human esophageal cancer cells by targeting CDK4.

Key words: MiR-124; Radiotherapy; Esophageal cancer; CDK4

\section{INTRODUCTION}

Esophageal cancer is one of the most common cancers, and is ranked eighth in incidence and sixth in mortality worldwide (Tang et al., 2014). The 5-year survival rate of postoperative esophageal cancer increased to $25 \%$ with the adoption of precise radiotherapy with chemotherapy (Rousseau et al., 2013). Thus, a better understanding of the molecular mechanisms of esophageal cancer is critical to improve the effectiveness of radiation treatment.

Recent studies have shown that some microRNAs (miRNA or miR) are dysregulated in several types of cancer and can contribute to tumor proliferation and resistance to radiotherapy (Weidhaas et al., 2007; Ma et al., 2012; Zhao et al., 2013). miRNAs are a class of small ( 22 nucleotides) non-coding RNA molecules that regulate gene expression via targeting mRNA for degradation or inhibition of translation (Hauptman and Glavac, 2013). miRNAs exert their gene regulatory activity primarily by imperfect base pairing to the 3' UTR of their target mRNAs (Finnegan and Matzke, 2003). As regulators of mRNA translation, miRNAs are involved in many biological processes including developmental timing, stem cell function, cellular differentiation, proliferation, apoptosis, cell cycle, and diseases such as cancer (Shin et al., 2009; Cellini et al., 2014). Furthermore, many studies have explored the association between miRNAs and radiosensitivity of cancer cells (Liu et al., 2013b; Yuan et al., 2014).

miR-124 is the most abundant miRNA in adult and embryonic central nervous system (Visvanathan et al., 2007). Previous studies showed that the expression level of miR-124 was significantly decreased in glioma, colorectal cancer, breast cancer, prostate cancer, and bladder cancer (Deng et al., 2013; Chen et al., 2014; Zhang et al., 2014b; Zhang et al., 2014c; Feng et al., 2015), where it acts as a tumor suppressor. Additionally, recent studies have proven that miR124 can radiosensitize glioma and colorectal cancer through the miR-124/cyclin-dependent kinases (CDK) 4 axis (Deng et al., 2013; Zhang et al., 2014c). CDKs play important roles in the control of cell division and modulate transcription in response to several extra- and intracellular cues. CDK4 is a critical member of the cell-cycle-related subfamilies (Malumbres, 2014), which have been reported to participate in a variety of physiological processes including cell proliferation and apoptosis (Zhang et al., 2014b; Feng et al., 2015). These studies made us speculate that miR-124 might function as a radiosensitizer in esophageal cancer, and the CDK4 signaling pathway might be involved in these radiosensitizing effects.

In this study, we aimed to determine the role of miR-124 in radiotherapy sensitivity in esophageal cancer. First, we explored the expression of miR-124 in 18 paired esophageal cancer and adjacent non-cancerous tissues, and then characterized the function of miR-124 in cell proliferation, invasion, and apoptosis. Furthermore, in order to identify the mechanisms involved in miR-124-mediated effects, the luciferase reporter gene assay was performed to confirm that CDK4 is a direct target of miR-124. 


\section{MATERIAL AND METHODS}

\section{Tissue specimens}

Eighteen pairs of specimens of primary esophageal cancer and adjacent non-tumor esophageal tissues were obtained from consecutive patients who underwent primary surgical resection of esophageal cancer between 2013 and 2014 at Taixing People's Hospital (China). The matched normal tissues were obtained from a $5 \mathrm{~cm}$ distance from the tumor margin, which was further confirmed by pathologists. All patients did not receive any therapy before recruitment to this study. The use of the tissue samples for all experiments were obtained with patient consent and approved by the Research Ethics Committee of Bengbu Medical School (China).

\section{Cell lines and culture conditions}

Esophageal cancer cell line TE-1 was purchased from Cell Bank of Chinese Academy of Sciences (Shanghai, China). These cells were maintained in RPMI 1640 medium (Gibco, Invitrogen, Carlsbad, CA, USA) supplemented with 10\% fetal bovine serum (Gibco), $100 \mathrm{U} /$ $\mathrm{mL}$ penicillin, and $100 \mathrm{mg} / \mathrm{mL}$ streptomycin (Beyotime Biotech, Haimen, China) in humidified air at $37^{\circ} \mathrm{C}$ with $5 \% \mathrm{CO}_{2}$.

\section{Cell transfection}

The miR-124 mimics and the negative control (NC) miRNA were synthesized by GenePharma (Shanghai, China) and transfected into TE-1 cells to a final oligonucleotide concentration of $30 \mathrm{nM}$. All cell transfections were performed with Lipofectamine ${ }^{\mathrm{TM}} 2000$ reagent (Invitrogen) according to the manufacturer instructions. For each treatment group, the experiments were performed in triplicate. Cells were collected for further experiments $48 \mathrm{~h}$ post-transfection.

\section{RNA isolation, reverse transcription (RT), and quantitative real-time polymerase chain reaction (PCR)}

Total RNA was extracted from the cells using TRIzol Reagent (Invitrogen). cDNA was synthesized using the PrimeScript ${ }^{\mathrm{TM}}$ RT reagent Kit (Takara, Dalian, China). A quantitative RT-PCR was carried out to detect the expression of mature miR-124 using SYBR Green qPCR kit (Thermo Scientific, Waltham, MA, USA) using a Bio-Rad CFX96 Real-Time PCR system(Bio-Rad, Tacoma, WA, USA). U6 snRNA was used as the endogenous control. The sequences of primers are shown in Table 1.

\section{Ionizing radiation (IR)}

TE-1 cells were seeded at a predetermined number of cells into a 6-well plate and transfected with miR-124 mimics (30 nM) or negative control miRNA (30 nM). After $24 \mathrm{~h}$ incubation, cells were treated with X-ray IR from a $6 \mathrm{MeV}$ linear accelerator (Taixing People's Hospital, Taixing, China). 


\section{Table 1. Primer sequences used in this study.}

\begin{tabular}{l|l|l}
\hline Name & Primers & \\
\hline \multirow{2}{*}{ miR-124 } & Reverse transcription & 5'-GTCGTATCCAGTGCAGGGTCCGAGGTATTCGCACTGGATACGACGGCATT-3' \\
\cline { 2 - 3 } & Forward primer & 5'-GCTAAGGCACGCGGT-3' \\
\cline { 2 - 3 } & Reverse primer & 5'-GTGCAGGGTCCGAGG-3' \\
\hline \multirow{2}{*}{ U6 } & Reverse transcription & 5'-AAAATATGGAACGCTTCACGAATTTG-3' \\
\cline { 2 - 3 } & Forward primer & 5'-CTCGCTTCGGCAGCACATATACT-3' \\
\cline { 2 - 3 } & Reverse primer & 5'-ACGCTTCACGAATTTGCGTGTC-3' \\
\hline
\end{tabular}

\section{Cell counting kit-8 (CCK-8) assay}

After treatment with miRNA, TE-1 cells were seeded in a 96-well plate at a density of $4 \times 10^{3}$ cells/well and incubated for $48 \mathrm{~h}$. In vitro cell proliferation was measured using the CCK-8 kit (Beyotime Biotech, Haimen, China) according to manufacturer instructions. The absorbance of each well at $450 \mathrm{~nm}\left(630 \mathrm{~nm}\right.$ reference) was determined by iMark ${ }^{\mathrm{TM}}$ microplate absorbance reader (Bio-Rad, Hercules, CA, USA). Each experiment was repeated in triplicate.

\section{Wound healing assay}

Cells were grown to near $100 \%$ confluence in 6-well plates. Twenty-four hours after transfection, linear scratch wounds were created on the confluent cell monolayer using a $100-\mu \mathrm{L}$ pipette tip. To stop cells from entering the cell cycle prior to wounding, cells were maintained in serum-free medium. To visualize migrating cells and wound healing, images were taken at 0,12 , and $24 \mathrm{~h}$ after scratching. A total of ten areas were selected randomly from each well and the cells in three wells of each group were quantified. Three independent experiments were performed.

\section{Hoechst 332528 staining}

Following experimental treatment, TE-1 cells were stained with Hoechst 332528 (5 $\mathrm{mg} / \mathrm{mL}$; Beyotime Biotech) for $10 \mathrm{~min}$ in the dark, and then washed extensively three times with PBS. Nuclear staining was examined under an Olympus IX73 Inverted Fluorescence Microscope (Olympus Optical Co. Ltd., Tokyo, Japan).

\section{Luciferase reporter assay}

The 3' UTR segment of CDK4 containing the predicted miR-124 target site was amplified by PCR from human genomic DNA. The 3' UTR segment of CDK4 with the mutant miR-124 target site was generated by a Quik Change site-directed mutagenesis kit (Stratagene, La Jolla, CA, USA). A total of $1 \times 10^{4}$ cells were seeded per well in $150 \mu \mathrm{L}$ RPMI 1640 medium. A total of $100 \mathrm{ng}$ wild-type (wild) or mutant (mut) CDK4 3' UTR reporter constructs were co-transfected with Lipofectamine ${ }^{\mathrm{TM}} 2000$ transfection reagent into TE-1 cells with 30 $\mathrm{nM}$ miR-124 or NC-miRNA according to the manufacturer instructions. After incubating the cells for $48 \mathrm{~h}$, the luciferase activity assay was performed with the Dual Luciferase reporter assay system (Promega Corp., Madison, WI, USA). The activity of firefly luciferase was measured and normalized to the corresponding Renilla luciferase activity. 


\section{Western blot analysis}

Proteins were separated by SDS-PAGE and transferred to polyvinylidene difluoride (PVDF) membranes (Millipore, Bedford, MA, USA). The membrane was blocked with blocking buffer (Beyotime) and incubated with the mouse anti-CDK4 monoclonal antibody (1:1000; Abcam, UK) and the mouse anti-GAPDH monoclonal antibody $(1: 2000$; Beyotime $)$ at $4{ }^{\circ} \mathrm{C}$ overnight. The secondary antibody incubations were performed for $2 \mathrm{~h}$ at room temperature and the proteins were detected with enhanced chemiluminescence reagents (Beyotime).

\section{Statistical analysis}

Data ware presented as the mean \pm standard deviation (SD) of three independent experiments. Statistical package for the Social Sciences for Windows Version 12.0 (SPSS, Chicago, IL, USA) was used to conduct all of the relative analyses using either the Student $t$-test or two-way ANOVA. $\mathrm{P}<0.05$ was considered statistically significant. All graphs were generated with GraphPad Prism Version 5.0 (GraphPad, La Jolla, CA, USA).

\section{RESULTS}

\section{miR-124 expression is suppressed in esophageal cancer tissues}

In an attempt to explore the expression and significance of miR-124 in esophageal cancer, we first detected its expression in 18 pairs of esophageal cancer tissues and adjacent normal tissues. Real-time PCR assay revealed that miR-124 expression was markedly decreased in esophageal cancer tissues compared with the adjacent noncancerous tissues (Figure 1). Collectively, these results suggest that miR-124 is downregulated in esophageal cancer tissues.

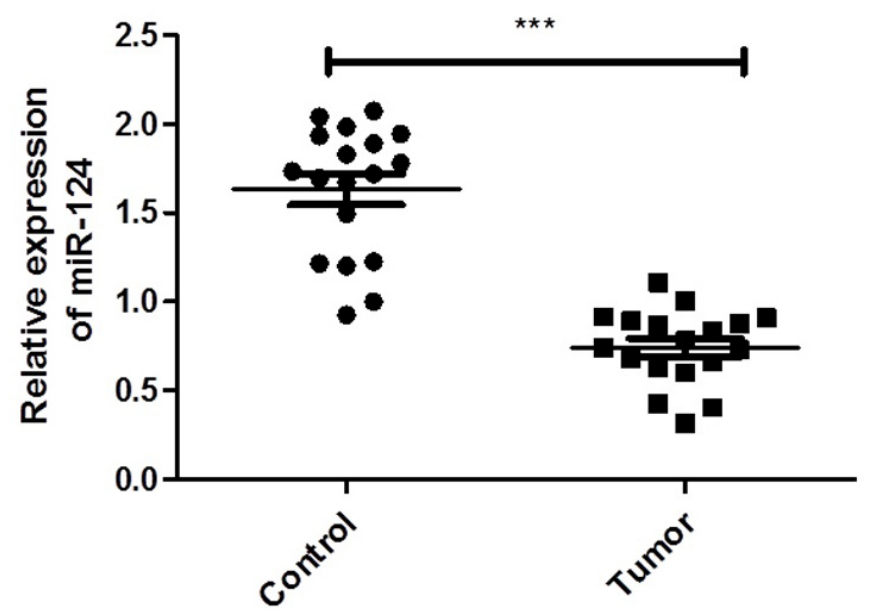

Figure 1. miR-124 expression in esophageal cancer tissues. Relative expression of miR-124 in 18 esophageal cancer tissues (tumor) compared with their pair-matched adjacent non-tumor tissues (control). miR-124 was detected by qRT-PCR and normalized to U6 levels. Data are reported as individual samples $(\mathrm{N}=18)$ with the line indicating the mean, $* * * \mathrm{P}<0.001$. 


\section{miR-124 inhibited the growth and invasion of the esophageal cancer cell TE-1}

To investigate the effect of miR-124 on cell growth and invasion, we transfected the esophageal cancer cell line TE-1 with miR-124 mimics. The average transducing units per cell give $85 \%$ or greater transfection efficiencies (Figure $2 \mathrm{~A}$ ). Upon transfection, the intracellular level of miR-124 was 93-fold higher in TE-1 cells (Figure 2B). CCK-8 and wound healing assays showed that ectopic expression of miR-124 could markedly inhibit the proliferation and invasion of TE-1 cells compared with the NC-mimic control (Figure 2C, 2D and 2E).

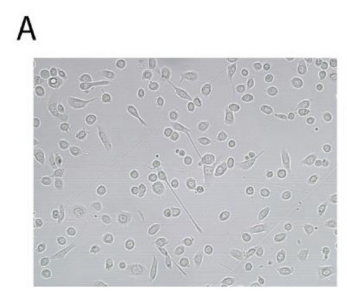

C

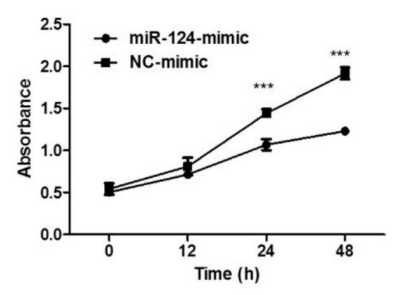

$E$
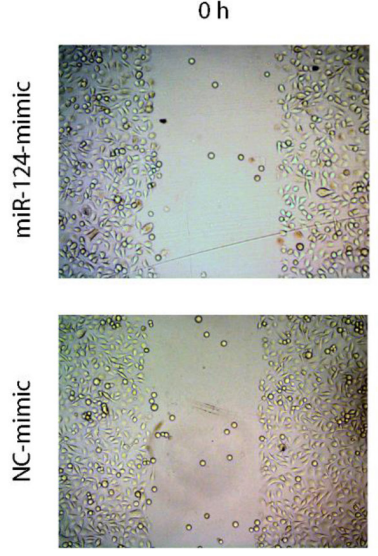

B
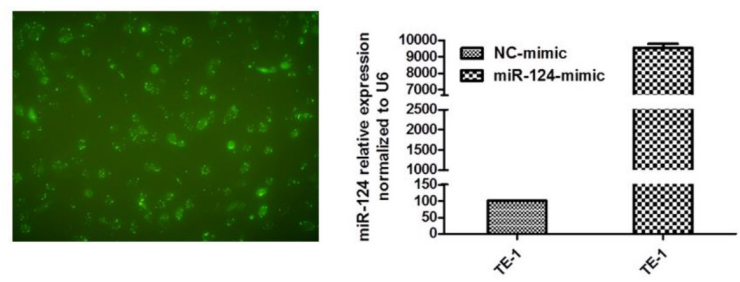

D

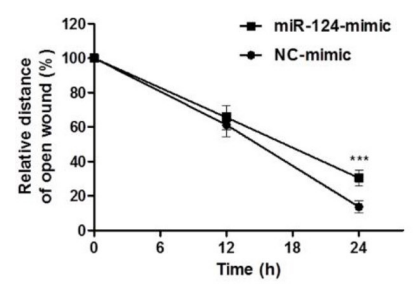

$12 \mathrm{~h}$
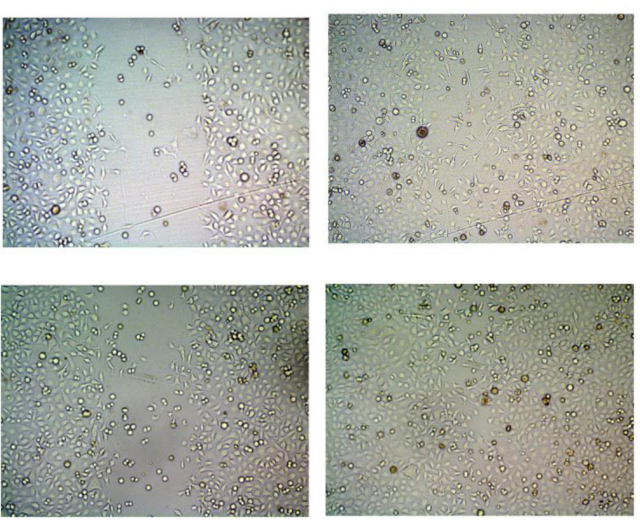

Figure 2. Overexpression of miR-124 inhibited the growth and invasion of esophageal cancer cell line TE-1. A. Fluorescence in cells was shown to be $85 \%$ (transducing units per cell) after transfection with miR-124 mimic. Bright field (left) and green fluorescence (right) are shown. B. RT-PCR was performed to detect the expression of miR-124 in esophageal cancer cell line TE-1 after treatment with miR-124 mimic or NC-miRNA (normalized to U6). C. CCK-8 assay was used to detect the effect of miR-124 and NC-miRNA on cell proliferation of TE-1. The results are reported as means $\pm \mathrm{SD}$ of the optical density (OD) values obtained in 3 independent experiments, $* * * \mathrm{P}<0.001$. D. E. Effects of invasion using a wound healing assay after treatment with miR-124 mimic or NCmiRNA. Bright field microscopy of cells are shown at 0,12 and $24 \mathrm{~h}$ post-wound induction in $\mathbf{E}$ and the results are quantitated in $\mathbf{D}, * * * \mathrm{P}<0.001$. 


\section{Elevated miR-124 expression confers radiosensitivity in esophageal cancer cells}

To investigate whether miR-124 increases radiosensitivity in esophageal cancer, TE-1 cells were transfected with miR-124 mimics or NC-miRNA using Lipofectamine ${ }^{\mathrm{TM}} 2000$. Twenty-four hours after transfection, the cells were exposed to 8 Gy (6 MV X-ray) of IR. According to the result of the CCK-8 assay, ectopic expression of miR-124 led to a higher percentage of apoptotic cells (Figure 3A). Similar results were obtained in the Hoechst 332528 stain assay (Figure 3B).

\section{A}
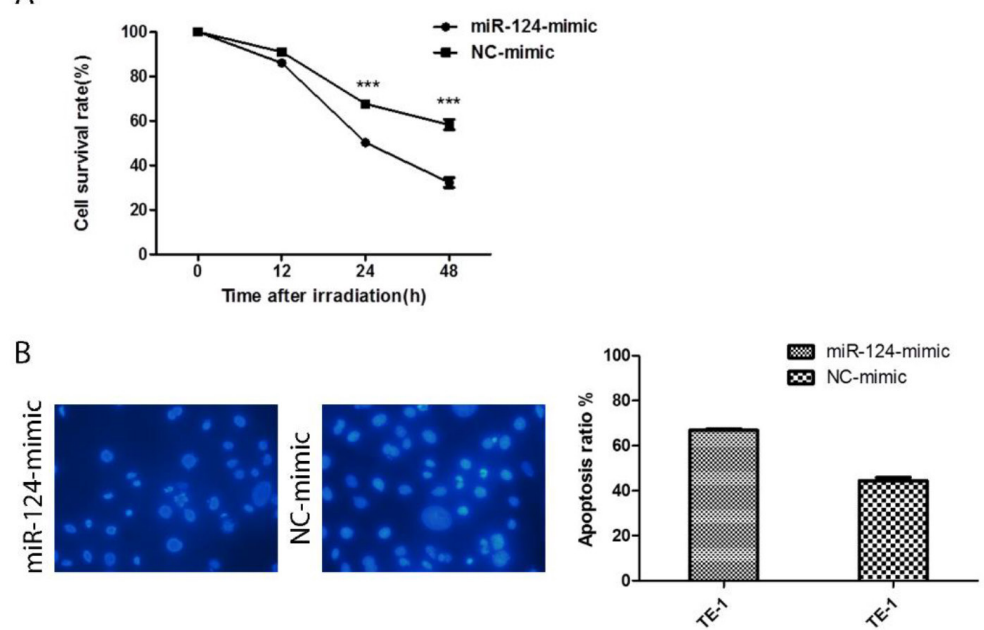

Figure 3. miR-124 enhanced the apoptosis of TE-1 cells induced by IR. A. CCK-8 assay was used to detect the effects of miR-124 and NC-miRNA on cell radiosensitivity, ${ }^{* * *} \mathrm{P}<0.001$. B. Forty-eight hours after radiotherapy, Hoechst 332528 stain assay was used to measure the apoptosis of TE-1 cells after treatment with miRNA-124 or NC-miRNA. Visualization of the stain is shown in the left panels and the apoptosis ratio in the right panel.

\section{CDK4 is the direct target of miR-124}

It is well known that miRNAs execute post-transcriptional regulation by binding to the 3' UTR of mRNAs. In order to elucidate the molecular mechanisms by which miR124 enhances the apoptosis of esophageal cancer cells through IR, we used the online tool TargetScanHuman 6.2 (http://www.targetscan.org/) to investigate the potential targets of miR-124. Subsequently, CDK4 was selected as the main target of miR-124 (Figure 4A). To find out if miR-124 directly binds to the 3' UTR of CDK4, the luciferase reporter gene assay was performed. TE-1 cells were co-transfected with luciferase reporter gene plasmids and miR-124 or NC-miRNA. Compared to NC-miRNA, miR-124 mimics significantly inhibited the luciferase activity of the reporter gene containing the wild-type 3' UTR of CDK4, but did not affect the luciferase activity of the reporter gene containing the mutant 3' UTR of CDK4 (Figure 4B). In addition, western blot confirmed that overexpression of miR-124 decreased CDK4 protein levels (Figure 4C). Taken together, these findings suggest that CDK4 is a direct target of miR-124. 
A

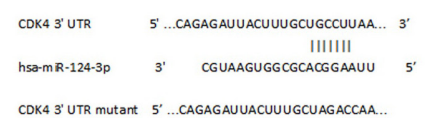

B

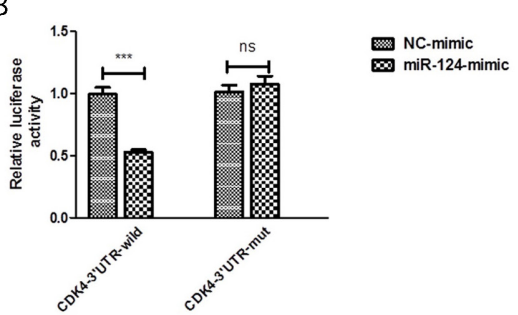

C

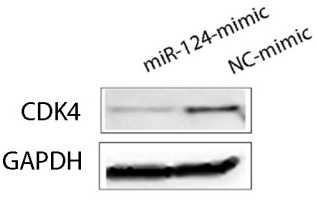

Figure 4. CDK4 is the direct target of miR-124. A. Binding sites for miR-124 and CDK4 3' UTR. Mutation of the 3' UTR in CDK4 prevents binding of miR-124. B. Relative luciferase activity of the indicated CDK4 reporter construct (wild-type or mutant) in TE-1 cell lines, co-transfected with miR-124 mimic or NC- mimic, are shown. C. Western blot analysis showed miR-124 transfection (but not NC-miRNA) reduced CDK4 protein levels.

\section{DISCUSSION}

Esophageal carcinoma is one of the most common malignant tumors of the digestive system, with high rates of incidence and mortality, especially in China (Tang et al., 2014). Radiotherapy is an essential therapy for the patients with inoperable and locally advanced esophageal cancer (Shridhar et al., 2013). However, a large portion of esophageal cancer tumors develop resistance to radiotherapy so its effect in these patients is limited. To improve this situation, it is importance to enhance the radiation sensitivity of esophageal cancers. Recent studies have demonstrated that the process of radioresistance involves multiple molecular mechanisms (Halimi et al., 2012; Qian et al., 2014; Zhang et al., 2014a), and numerous studies suggest that miRNAs played an important role in response to ionizing radiation (Cellini et al., 2014). Previous studies have suggested that miRNAs can act as tumor suppressors by targeting crucial genes (Liu et al., 2013a,b; Yongchun et al., 2014). Of these, miR-124 has been reported to be important in a variety of cancers (Cao et al., 2007; Visvanathan et al., 2007). Wen et al. (2015) reported that miR-21 might be involved in the development and metastasis of esophageal cancer through its interaction with target genes programmed cell death 4 (PDCD4) and K-ras. Deng et al. (2013) showed that overexpression of CDK4 results in a poorer prognosis and confers resistance of glioma cells to radiation. Consequently, miR-124 could resensitize glioma cells by targeting CDK4. This is consistent with a report by Zhang et al. (2014c) who illustrated that miR-124 could increase the radiosensitivity of conditionally reprogrammed cells by blocking the expression of paired related homeobox 1 (PRRX1). It has also been shown that miR-124 expression is consistently suppressed in esophageal cancer tissues and cell lines, where it functions as a tumor suppressor (Cheng et al., 2015). Similar to their results, we found that the expression of miR-124 was downregulated in esophageal cancer tissues.

In functional studies, reintroduction of miR-124 markedly restrained proliferation and invasion of TE-1 cells. Additionally, ectopic expression of miR-124 could enhance apoptosis of esophageal cancer cells induced by ionizing radiation. These results suggest that miR-124 plays a role in proliferation and invasion, and might act as a radiosensitizer in esophageal cancer cells. 
miR-124 radiosensitizes human esophageal cancer

Although several molecular alterations were identified in esophageal cancer, such as the miR-124/STAT3 axis, the miR-124/CDK4 axis is of particular importance. miR-124/ CDK4 was also reported as a tumor suppressor in breast cancer and plays an important role in radiosensitizing human colorectal cancer cells (Deng et al., 2013; Feng et al., 2015). CDK4 is a key protein involved in cell cycle, and complexes between D-type cyclins (D1, D2, D3) and CDK4 or CDK6 phosphorylate and inactivate retinoblastoma protein (Rb) (Rubin, 2013). This results in derepression of multiple genes encoding proteins required for DNA synthesis (S phase) or mitosis (Malumbres and Barbacid, 2001). Transition through G1 to $\mathrm{S}$ phase is controlled by the regulatory subunits D-type cyclins (CDK4 and CDK6) and the CDK inhibitor p16 (INK4a) (Wu et al., 2011). Previous studies have demonstrated that CDK4 is an unfavorable prognostic factor, as it mediates cell cycle progression (Wu et al., 2011; Zhang et al., 2014b; Feng et al., 2015). Furthermore, it was observed that upregulation of CDK4 is associated with radiotherapy resistance in human glioma cells (Deng et al., 2013). In this study, we investigated the role of miR-124 in regulating radiosensitivity of esophageal cancer cells and demonstrated that CDK4 is the direct target of miR-124. Western blot assay confirmed that overexpression of miR-124 decreased CDK4 protein levels. Based on these observations, we hypothesize that the downregulation of CDK4 simultaneously weakens the self-renewal properties of cells, both of which are closely related to radiotherapy resistance.

In conclusion, our results confirm a functional link between miR-124 and CDK4 expression in esophageal cancer, indicating that CDK4 is the direct target of miR-124, which subsequently inhibits its downstream signaling pathway. Increased expression of miR-124 will lead to lower expression levels of CDK4, which enhances apoptosis of esophageal cancer cells induced by ionizing radiation. Based on these data, we suggest that miR-124 and CDK4 are promising radiosensitivity-associated targets in the treatment of esophageal cancer and further studies are warranted to examine these targets for treatment and therapy.

\section{Conflicts of interest}

The authors declare no conflict of interest.

\section{ACKNOWLEDGMENTS}

Research supported by Taixing People's Hospital (Program \#try1518).

\section{REFERENCES}

Cao X, Pfaff SL and Gage FH (2007). A functional study of miR-124 in the developing neural tube. Genes Dev. 21: 531536. http://dx.doi.org/10.1101/gad.1519207

Cellini F, Morganti AG, Genovesi D, Silvestris N, et al. (2014). Role of microRNA in response to ionizing radiations: evidences and potential impact on clinical practice for radiotherapy. Molecules 19: 5379-5401. http://dx.doi. org/10.3390/molecules 19045379

Chen J, Xiao H, Huang Z, Hu Z, et al. (2014). MicroRNA124 regulate cell growth of prostate cancer cells by targeting iASPP. Int. J. Clin. Exp. Pathol. 7: 2283-2290.

Cheng Y, Li Y, Nian Y, Liu D, et al. (2015). STAT3 is involved in miR-124-mediated suppressive effects on esophageal cancer cells. BMC Cancer 15: 1-22. http://dx.doi.org/10.1186/s12885-015-1303-0

Deng X, Ma L, Wu M, Zhang G, et al. (2013). miR-124 radiosensitizes human glioma cells by targeting CDK4. $J$. Neurooncol. 114: 263-274. http://dx.doi.org/10.1007/s11060-013-1179-2 
Feng T, Xu D, Tu C, Li W, et al. (2015). MiR-124 inhibits cell proliferation in breast cancer through downregulation of CDK4. Tumour Biol. 36: 5987-5997. http://dx.doi.org/10.1007/s13277-015-3275-8

Finnegan EJ and Matzke MA (2003). The small RNA world. J. Cell Sci. 116: 4689-4693. http://dx.doi.org/10.1242/ jes. 00838

Halimi M, Asghari SM, Sariri R, Moslemi D, et al. (2012). Cellular response to ionizing radiation: a MicroRNA story. Int. J. Mol. Cell. Med. 1: 178-184.

Hauptman N and Glavac D (2013). MicroRNAs and long non-coding RNAs: prospects in diagnostics and therapy of cancer. Radiol. Oncol. 47: 311-318.

Liu YJ, Lin YF, Chen YF, Luo EC, et al. (2013a). MicroRNA-449a enhances radiosensitivity in CL1-0 lung adenocarcinoma cells. PLoS One 8: e62383. http://dx.doi.org/10.1371/journal.pone.0062383

Liu ZL, Wang H, Liu J and Wang ZX (2013b). MicroRNA-21 (miR-21) expression promotes growth, metastasis, and chemo- or radioresistance in non-small cell lung cancer cells by targeting PTEN. Mol. Cell. Biochem. 372: 35-45. http://dx.doi.org/10.1007/s11010-012-1443-3

Ma R, Jiang T and Kang X (2012). Circulating microRNAs in cancer: origin, function and application. J. Exp. Clin. Cancer Res. 31: 38-46. http://dx.doi.org/10.1186/1756-9966-31-38

Malumbres M (2014). Cyclin-dependent kinases. Genome Biol. 15: 122-131.http://dx.doi.org/10.1186/gb4184

Malumbres M and Barbacid M (2001). To cycle or not to cycle: a critical decision in cancer. Nat. Rev. Cancer 1: 222-231. http://dx.doi.org/10.1038/35106065

Qian D, Zhang B, Zeng XL, Le Blanc JM, et al. (2014). Inhibition of human positive cofactor 4 radiosensitizes human esophageal squmaous cell carcinoma cells by suppressing XLF-mediated nonhomologous end joining. Cell Death Dis. 5: e1461.http://dx.doi.org/10.1038/cddis.2014.416

Rousseau D, Capitain O, Denis F, Girault S, et al. (2013). [Esophageal cancer: outcome according to therapeutic strategy]. Cancer Radiother. 17: 10-20. http://dx.doi.org/10.1016/j.canrad.2012.10.011

Rubin SM (2013). Deciphering the retinoblastoma protein phosphorylation code. Trends Biochem. Sci. 38: 12-19. http:// dx.doi.org/10.1016/j.tibs.2012.10.007

Shin S, Cha HJ, Lee EM, Lee SJ, et al. (2009). Alteration of miRNA profiles by ionizing radiation in A549 human nonsmall cell lung cancer cells. Int. J. Oncol. 35: 81-86.

Shridhar R, Almhanna K, Meredith KL, Biagioli MC, et al. (2013). Radiation therapy and esophageal cancer. Cancer Contr. 20: 97-110.

Tang WR, Fang JY, Wu KS, Shi XJ, et al. (2014). Epidemiological characteristics and prediction of esophageal cancer mortality in China from 1991 to 2012. Asian Pac. J. Cancer Prev. 15: 6929-6934. http://dx.doi.org/10.7314/ APJCP.2014.15.16.6929

Visvanathan J, Lee S, Lee B, Lee JW, et al. (2007). The microRNA miR-124 antagonizes the anti-neural REST/SCP1 pathway during embryonic CNS development. Genes Dev. 21: 744-749. http://dx.doi.org/10.1101/gad.1519107

Weidhaas JB, Babar I, Nallur SM, Trang P, et al. (2007). MicroRNAs as potential agents to alter resistance to cytotoxic anticancer therapy. Cancer Res. 67: 11111-11116. http://dx.doi.org/10.1158/0008-5472.CAN-07-2858

Wen SW, Zhang YF, Li Y, Liu ZX, et al. (2015). Characterization and effects of miR-21 expression in esophageal cancer. Genet. Mol. Res. 14: 8810-8818.http://dx.doi.org/10.4238/2015.August.3.4

Wu A, Wu B, Guo J, Luo W, et al. (2011). Elevated expression of CDK4 in lung cancer. J. Transl. Med. 9: 38-46. http:// dx.doi.org/10.1186/1479-5876-9-38

Yongchun Z, Linwei T, Xicai W, Lianhua Y, et al. (2014). MicroRNA-195 inhibits non-small cell lung cancer cell proliferation, migration and invasion by targeting MYB. Cancer Lett. 347: 65-74. http://dx.doi.org/10.1016/j. canlet.2014.01.019

Yuan W, Xiaoyun H, Haifeng Q, Jing L, et al. (2014). MicroRNA-218 enhances the radiosensitivity of human cervical cancer via promoting radiation induced apoptosis. Int. J. Med. Sci. 11: 691-696. http://dx.doi.org/10.7150/ijms.8880

Zhang P, Wang L, Rodriguez-Aguayo C, Yuan Y, et al. (2014a). miR-205 acts as a tumour radiosensitizer by targeting ZEB1 and Ubc13. Nat. Commun. 5: 5671-5680.http://dx.doi.org/10.1038/ncomms6671

Zhang T, Wang J, Zhai X, Li H, et al. (2014b). MiR-124 retards bladder cancer growth by directly targeting CDK4. Acta Biochim. Biophys. Sin. (Shanghai) 46: 1072-1079. http://dx.doi.org/10.1093/abbs/gmu105

Zhang Y, Zheng L, Huang J, Gao F, et al. (2014c). MiR-124 Radiosensitizes human colorectal cancer cells by targeting PRRX1. PLoS One 9: e93917. http://dx.doi.org/10.1371/journal.pone.0093917

Zhao L, Lu X and Cao Y (2013). MicroRNA and signal transduction pathways in tumor radiation response. Cell. Signal. 25: 1625-1634.http://dx.doi.org/10.1016/j.cellsig.2013.04.004 